\title{
Quantum degeneracy of microcavity polaritons
}

\author{
A. Baas, J.-Ph. Karr, M. Romanelli, A. Bramati, and E. Giacobino ${ }^{1}$ \\ ${ }^{1}$ Laboratoire Kastler Brossel, Université Paris 6, École Normale Supérieure et CNRS, \\ UPMC Case 74, 4 place Jussieu, 75252 Paris Cedex 05, France
}

\begin{abstract}
We investigate experimentally one of the main features of a quantum fluid constituted by exciton polaritons in a semiconductor microcavity, that is quantum degeneracy of a macroscopic fraction of the particles. We show that resonant pumping allows to create a macroscopic population of polaritons in one quantum state. Furthermore we demonstrate that parametric polariton scattering results in the transfer of a macroscopic population of polariton from one single quantum state into another one. Finally we briefly outline a simple method which provides direct evidence of the first-order spatial coherence of the transferred population.
\end{abstract}

In the past few years, the nature of quantum state of carriers in semiconductors and more specifically the possibility of condensation has raised a growing interest 1]. Exciton polaritons in semiconductor microcavities appear as promising candidates because they have a very small effective mass around the minimum energy state at $\mathbf{k}=\mathbf{0}$ (where $\mathbf{k}$ is the wave vector in the plane of the layers), providing a large critical temperature for condensation [2, 3]. Bosonic stimulation of the relaxation towards the bottom of the dispersion curve under nonresonant pumping has been reported by several groups [4, 5. In order to demonstrate condensation, it is crucial to give evidence of quantum degeneracy of polaritons. Here, we investigate this property in a very different situation, where the lower polariton branch is pumped by a resonant laser beam. The system is then far from thermodynamical equilibrium; the polariton oscillation frequency is not fixed by an equation of state relating the chemical potential to the polariton density and no condensation is expected to take place. The exciton polariton system is however a quantum fluid which exhibits collective excitations [ $[$ ] .

We show in this paper that a macroscopic fraction of the particles in the system are in one single quantum state. To our knowledge, there has been to date no complete experimental evidence of a polariton system being in a single quantum state. Such evidence is necessarily related to coherence properties of the polariton field, which are transferred to the emitted light field, due to its part-matter, part-light nature [7, 8]; this feature allows experimental access to the polariton coherence through measurements on the emitted light field. Most experimental studies have focused on the linewidth, angular divergence and phase coherence of the nonlinear emission 9]. Second-order coherence in the time domain has been studied theoretically [8, 10, 11] and experimentally through the measurement of the intensity autocorrelation function $g^{(2)}(\tau)$ of the emitted light [12].

The reduction of the linewidth and angular divergence above threshold may indicate a reduction of the number of populated polariton modes. However, such properties, related to the first-order coherence, are not in themselves sufficient proofs of the single-mode nature of the polariton field. In the same way, a measurement of first-order temporal coherence does not allow to discriminate between the light from a single-mode laser and the light from a spectrally filtered thermal source [13]. In order to obtain a full characterization the measurement of the spatial second-order coherence properties is a crucial one. The aim of this paper is to give an unambiguous experimental signature of polariton quantum degeneracy in the regime of resonant pumping of the lower polariton branch, by studying the second-order spatial coherence. We use specific methods from quantum optics to characterize the modes of the emitted light, which are directly related to the properties of the modes of the polariton quantum fluid.

It has been shown recently that the measurement of intensity correlations in the transverse plane provides a criterion allowing to characterize unambiguously singlemode and multimode quantum states of light 14. In this paper, we apply this criterion to the light emitted by a polariton population around $\mathbf{k}=\mathbf{0}$, created by polariton pair scattering under resonant pumping of the lower polariton branch by a single-mode cw laser. Our results show that the emitted light is in a single-mode quantum state, giving a proof of the quantum degeneracy of polaritons. We also demonstrate first-order spatial coherence through the observation of interference fringes in the emitted field.

The microcavity sample is a high quality factor $2 \lambda$ cavity described in [15] with polariton linewidths in the 100$200 \mu \mathrm{eV}$ range. The pump laser is a single-mode tunable cw Ti:Saphir with a linewidth of $1 \mathrm{MHz}$, spatially filtered through an optical fiber [16] and focused on a $100 \mu \mathrm{m}$ spot (full width at half maximum of the gaussian distribution) with a divergence of $0.6^{\circ}$. All experiments are carried out with one $\sigma^{+}$polarized resonant pump beam and no

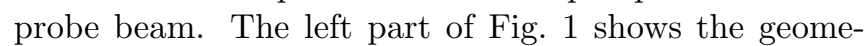
try of the experiment. Images of the nonlinear emission around $\mathbf{k}=\mathbf{0}$ are taken by CCD cameras in real-space (RS) (near field) and in $\mathbf{k}$-space (KS) (far-field).

We first consider the simplest case where the pump beam is at normal incidence and directly creates polari- 
tons around $\mathbf{k}=\mathbf{0}$. The interest of investigating this configuration is to check the widely used assumption that a resonant pump field creates polaritons in a single quantum state, provided the spot size and the pump divergence obey certain conditions. The recent observation of complex transverse patterns in the emitted light in the nonlinear regime 16, 17] raises some doubts about the single-mode character of the polariton population. Transverse intensity correlation measurements allow to answer this question unambiguously. From a practical point of view, it is not easy to analyze the properties of the polariton emission since it is in the same direction as the much more intense reflected laser field. However, at high enough excitation density the polaritons are confined by nonlinear effects in a zone that is smaller than the excitation spot 16] (see the RS image of the excitation spot in Fig. 2 a). Because of diffraction, the emitted field has a larger angular divergence than the reflected laser (see the KS image in Fig. 2 b). The reflected laser can then be filtered out and we perform the intensity correlation measurements on the remaining part of the emitted field.

Two methods will be used for the characterization of single-mode and multimode beams [14]:

(i) For a beam in a single-mode quantum state, a partial intensity measurement (i.e. only a part of the beam is sent to the photodetector) has the same effect as losses. This corresponds to the idea that in a single-mode quantum state, photons are randomly distributed in the transverse plane. Such a behavior can be tested by "cutting" the beam transversally with a razor blade and measuring the intensity noise as a function of intensity [18]. The noise normalized to the shot noise limit should vary linearly with the intensity -the slope is respectively positive, negative or equal to zero if the noise of the total beam is above, under or at the shot noise level. Any deviation from the linear behavior would prove the multimode character of the beam.

(ii) If two separate parts of the single-mode beam of equal intensities are sent to two identical photodetectors $A$ and $B$, the noise of the difference of the photocurrents $N_{A}-N_{B}$ is the shot noise, whatever the quantum state of the beam -even for a beam with excess noise, when the noise measured by each detector is above the shot noise. There are no correlations at the quantum level between $N_{A}$ and $N_{B}$, which again corresponds to the idea that photons are randomly distributed in the transverse plane. Any deviation from the shot noise level would prove the multimode character of the beam.

These methods only apply to the transverse singlemode or multimode nature of the beam. It is assumed that only one longitudinal cavity mode is present in the emission. For example, one can consider the case of a laser emitting on two consecutive longitudinal cavity modes, but with the same transverse distribution described by a $T E M_{00}$ mode; it is clear that the above-
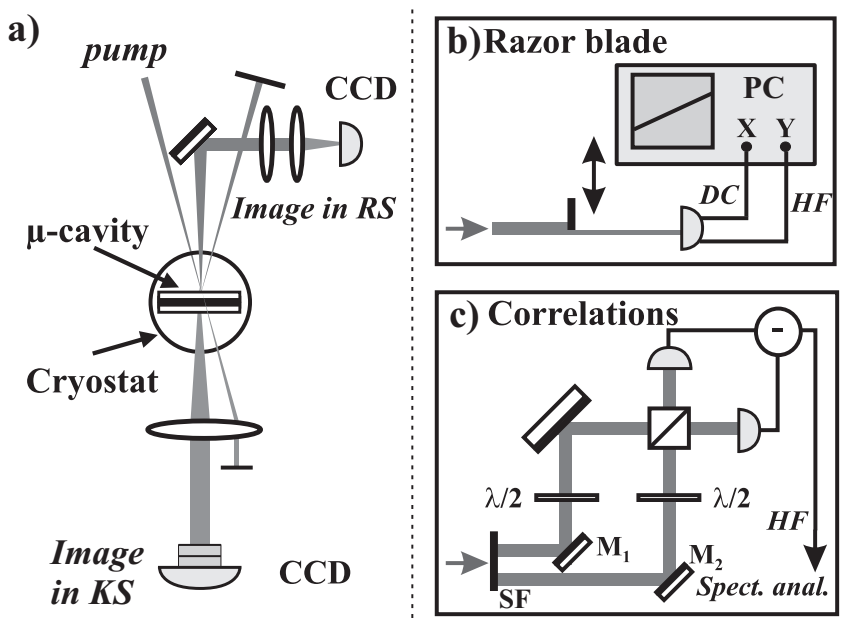

FIG. 1: a) Experimental setup. CCD camera for images in $\mathrm{RS}$ (KS) of the emitted light in reflection (transmission). b) "Razor blade" setup. HF (DC): high (low) frequency part of the signal. c) Intensity correlations set-up. Each of the mirrors $M_{1}$ and $M_{2}$ reflects the emission from one of the regions selected by the spatial filter (SF) in KS (see also Fig. 2b).

described experiments do not allow to evidence the multimode nature of such a beam. However in microcavities the emission is obviously longitudinal single-mode, because other longitudinal modes are very far in energy. In the same way it is assumed that only one polarization state is present in the emission. In our case, this condition can be reached by using a $\sigma^{+}$-polarized pump beam, since it was shown that the signal emission is then also $\sigma^{+}$polarized [19].

In these experiments, a multimode beam can be identified unambiguously by a deviation from the expected behavior for a single-mode beam. However, no necessary and sufficient condition can be given for the identification of a single-mode quantum state, because the single-mode behavior has to be verified in all transverse bases. But a convincing indication can be given if the single-mode behavior is verified in a basis of transverse modes in which the field can be expected to be multimode [14]. Since the polariton modes are well approximated by planes waves defined by an in-plane wave vector $\mathbf{k}$, the plane waves are a relevant basis to demonstrate the eventual multimode character of the emission; in the following, all measurements are done in the far field.

The curve in Fig. 3] a) shows the results of the "razor blade" experiment. The linear variation of the normalized noise as a function of intensity indicates the singlemode character of the emitted light ; the slope is positive because the beam has some excess noise.

This result was confirmed by the measurement of intensity correlations in the transverse plane. The experimental setup is shown in Fig 1 c). Two parts of the emitted beam are selected (as shown in Fig. 2 b) by placing a screen with two small holes in $\mathbf{k}$-space. The light 

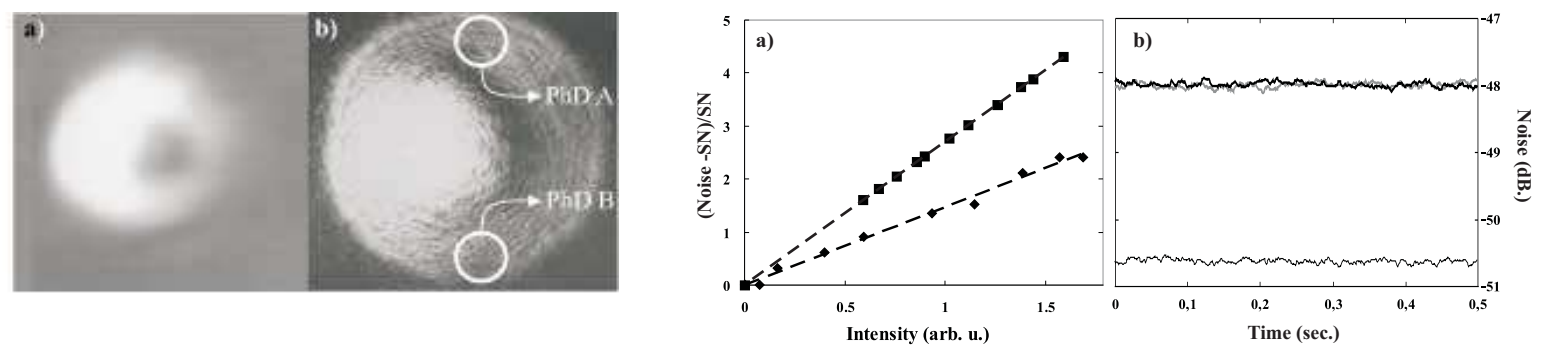

FIG. 3: a) Squares(diamonds): variations of the noise -HF signal- of the nonlinear emission around $\mathbf{k}=0$ as a function of the detected intensity -DC signal-, in the degenerate (non-degenerate) geometry. The shot noise (SN) is subtracted from the total noise, and the result is normalized to the SN. Cavity-exciton detuning: respectively 0.3 and $1.2 \mathrm{meV}$. In the degenerate case, points at very low intensity are missing because the noise was too small compared to dark noise. b) Noise power as observed on the screen of the spectrum analyzer. Dark line: noise of the intensity difference, perfectly superimposed with the shot noise level (gray line). Dark noise (clear gray line) is $2.6 \mathrm{~dB}$ below the $\mathrm{SN}$ level.

FIG. 2: Image of the non linear emission in RS (a) and in k-space (b) in the degenerate geometry [16]. Cavity exciton detuning $0.3 \mathrm{meV}$ and laser intensity $2.2 \mathrm{~mW}$. In (a) the dark region in the laser spot corresponds to the polariton resonance. In (b) the polariton emission is seen around the (brighter) reflected laser in $\mathbf{k}$-space, showing a larger angular divergence. The principle of the intensity correlation measurement is schematically indicated.

transmitted by each of the holes is sent to a detector by removing the $\lambda / 2$ waveplates and the high-frequency part of the photocurrent difference is sent to a spectrum analyzer. The polarizing cube, together with the $\lambda / 2$ waveplates, allows to measure the shot noise reference by separating each beam into two parts of equal intensities incident on each detector and making the difference of the photocurrents [20]. An example of our results is shown in Fig. 3 b). The noise of the intensity difference is the shot noise level, whereas each beam has an excess noise of more than $30 \%$. This experiment was repeated for different positions of the holes in $\mathbf{k}$-space, with identical results.

These results are a very strong indication that the emission around $\mathbf{k}=\mathbf{0}$ originates from one and the same polariton quantum state, i.e. the $\mathrm{k}=0$ state populated by resonant pumping. In particular, it implies that non- degenerate processes such as the pair scattering $\{\mathbf{0}, \mathbf{0}\} \rightarrow$ $\{\mathbf{k},-\mathbf{k}\}$ play a negligible role and validates the singlemode treatment adopted in [7, 11, 16, 17]. The larger divergence of the nonlinear emission with respect to that of the pump beam is due to diffraction effects and is not associated with a polariton population in other modes.

We now consider a pump at the so-called "magic angle" with a power slightly above the parametric oscillation threshold, where a bright signal beam is emitted around $\mathbf{k}=\mathbf{0}$ [21]. Also in this configuration, complex transverse patterns have been observed in the emitted light around $\mathbf{k}=\mathbf{0}$ [15, 22], raising questions about its single-mode character. Again, intensity correlation measurements provide an unambiguous answer.

Fig. [3 a) shows the result of a "razor blade"-type experiment (Fig. 1 b) on the light emitted around $\mathbf{k}=\mathbf{0}$ in reflection, the reflected pump beam being carefully filtered out. The emitted beam is cut by a razor blade in $\mathbf{k}$-space. The variation of the normalized noise as a function of the intensity is linear within experimental accuracy, the positive slope meaning that the total beam has excess noise. This experiment demonstrates that only one signal-idler pair oscillates, as can be expected from the analogy with optical parametric oscillators [23]. It validates the widely used model involving only three polariton $\mathbf{k}$ states [7], which successfully accounts for most experimental observations 2]. In other words, polariton pair scattering makes it possible to create a macroscopic population of polariton in one single quantum state distinct from the pumped mode. It would be very interesting to make the same kind of measurement below threshold - which requires more sensitive photodetectors than the one used here-, since many signal-idler pairs are then involved and evidence for a multimode quantum state 


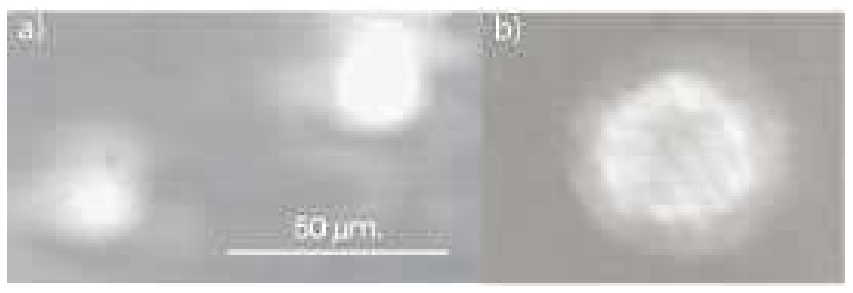

FIG. 4: Image of the light emitted in a cone of $2^{\circ}$ around the normal direction a)in $\mathrm{RS}$ in reflection ; b) in $\mathbf{k}$-space in transmission. Cavity-exciton detuning: $1.3 \mathrm{meV}$. Laser intensity: $2.8 \mathrm{~mW}$ (around 1.5 times the threshold intensity).

should be observed [24].

The single-mode nature of the signal polariton field implies phase coherence, i.e. first-order spatial coherence over the whole emission zone. In order to check this property, we use an original set-up directly revealing the phase coherence of the signal field. The idea is to superimpose the light fields emitted by two separate spots of the sample, sufficiently small to have a significant divergence so that the emitted fields overlap in $\mathbf{k}$-space. This is analogous to Young's double slit experiment, where the slits are put directly on the surface of the light source.

Fig. (4 a) shows a RS image of the sample surface, after filtration in KS of the light emitted in a cone of $2^{\circ}$ around the normal direction, which allows to get rid of the pump laser at the "magic angle" (about $12^{\circ}$ ). It can be seen that the signal field is emitted by two separate spots of a few microns in diameter and separated by $l \simeq$ $70 \mu \mathrm{m}$. The cavity-exciton detuning and excitation intensity have been chosen precisely to reach this situation , which is caused by the interplay between nonlinear and disorder effects, so as to make the implementation of our Young's experiment easier. However, the separation of the polariton field in two spots is not in principle necessary for the experiment; alternatively, it is possible to make an image of the surface of the sample and place two slits in the image plane.

The corresponding image in KS presents interference fringes (Fig. 目b). We are confident that they are not due to a self-interference of the laser, because the laser light has been filtered out. We sent the emitted field through a monochromator and found no component at the laser wavelength. Moreover, the fringes are perpendicular to the line joining the two spots and the fringe separation is in full agreement with a simple two-slits type calculation: $i=\lambda f / / l$, with $\mathrm{f}$ the focal of the lens. This experiment reveals a significant degree of first-order spatial coherence of the signal polaritons at a scale of $70 \mu \mathrm{m}$. The fact that interference is observed in cw regime confirms, in addition, that the beams emitted by the two spots originates from the same polariton mode (in spite of the complex shape of the active region in RS). Indeed, two distinct modes would interfere during a time limited by the coherence time $T_{2}$, in the same way as the emissions from two independent lasers 13 .

This result is in full agreement with the single-mode properties discussed above. It also illustrates a direct method of measurement of first-order spatial coherence and gives an order of magnitude for the coherence length of the polaritons created by the parametric process, which can be compared to the coherence length of polaritons in the linear regime 25].

We have presented an experimental study of quantum degeneracy of microcavity polaritons, relying on the measurement of intensity correlations in the transverse plane. We have shown that (i) resonant pumping allows to create a macroscopic population of polaritons in a single quantum state; (ii) in the case of non-degenerate interaction, the polariton pair scattering gives rise to a singlemode quantum state for the polariton population. These results are in agreement the description of the system as a quantum fluid in which only a few collective excitation modes are populated [6]. The experimental methods that we have used are very promising for the characterization of quantum degeneracy [5, 26], also under nonresonant pumping.

We acknowledge enlightening discussions with I. Carusotto, C. Ciuti, O. El Daïf, C. Fabre, T. Guillet, N. Treps, and J. Tignon. We are very grateful to R. Houdré for providing us with the microcavity sample.

[1] S. A. Moskalenko and D. W. Snoke, Bose-Einstein Condensation of Excitons and Biexcitons and Coherent Nonlinear Optics with Excitons (Cambridge University Press, Cambridge, 2000).

[2] Semicond. Sci. Technol. 18, S279-S434, special issue on semiconductor microcavities, edited by J. J. Baumberg and L. Vina (2003).

[3] Y. G. Rubo et al., Phys. Rev. Lett. 91, 156403 (2003); J. Keeling et al., Phys. Rev. Lett. 93, 226403 (2004).

[4] D. Le Si Dang et al., Phys. Rev. Lett. 81, 3920 (1998); P. Senellart et al., Phys. Rev. B 62, R16263 (2000); R. Butté et al., Phys. Rev. B 65, 205310 (2002).

[5] M. Richard et al., Phys. Rev. B 72, 201301 (2005).

[6] I. Carusotto and C. Ciuti,Phys. Rev. Lett. 93, 166401 (2004);

[7] C. Ciuti et al., Phys. Rev. B 62, R4825 (2000).

[8] J. Ph. Karr et al.,Phys. Rev. A 69, 063807 (2004).

[9] J. J. Baumberg et al., Phys. Rev. B 62, R16247 (2000); G. Messin et al., Phys. Rev. Lett. 87, 127403 (2001); A. Huynh et al., Phys. Rev. Lett. 90, 106401 (2003); S. Kunderman et al., Phys. Rev. Lett 91, 156403 (2003).

[10] F. Tassone and Y. Yamamoto, Phys. Rev. A 62, 063809 (2000); F. P. Laussy et al., Phys. Rev. Lett. 93, 016402 (2004); D. Sarchi and V. Savona, cond-mat/0411084

[11] P. Schwendimann et al., Phys. Rev. B 68, 165324 (2003).

[12] H. Deng et al., Science 298, 199 (2002).

[13] L. Mandel and E. Wolf, Optical Coherence and Quantum Optics (Cambridge University Press, Cambridge, 1995).

[14] M. Martinelli et al., Phys. Rev. A 67, 023808 (2003).

[15] R. Houdré et al., Phys. Rev. Lett. 85, 2793 (2000). 
[16] A. Baas et al., Phys. Rev. A 69, 023809 (2004).

[17] J. Ph. Karr et al., Phys. Rev. A 69, 031802(R) (2004).

[18] J.-Ph. Poizat et al., J. Opt. Soc. Am. B 15, 1757 (1998); J.-P. Hermier et al., J. Opt. Soc. Am. B 16, 2140 (1999).

[19] P. G. Lagoudakis et al., Phys. Rev. B 65, 161310 (2002).

[20] H.A. Bachor, A Guide to Experiments in Quantum Optics, Wiley, Weinheim, pp. 181-183 (1998).

[21] P. G. Savvidis et al., Phys. Rev. Lett. 84, 1547 (2000); R. M. Stevenson et al., Phys. Rev. Lett. 85, 3680 (2000); J. Erland et al., Phys. Rev. Lett. 86, 5791 (2001); M.
Saba et al. Nature, 414, 731 (2001); A. I. Tartakovskii et al., Phys. Rev. B 65, 081308 (2002).

[22] A. Baas et al., Phys. Rev. B 70 161307(R) (2004).

[23] C. Fabre et al., C. R. Acad. Sci. Paris 1 IV, 553 (2000).

[24] W. Langbein et al., Phys. Rev. B 70, 205301 (2004).

[25] A. I. Tartakovskii et al., Phys. Stat. Sol. B 221, 163 (2000).

[26] L. V. Butov et al., Nature 417, 47 (2003); C. W. Lai et al., Science 303, 503 (2004). 